Check for updates

Cite this: RSC Adv., 2019, 9, 7136

Received 19th December 2018

Accepted 15th February 2019

DOI: $10.1039 / c 8 r a 10415 h$

rsc.li/rsc-advances

\section{Enhancement effect of reduced graphene oxide and silver nanocomposite supported on poly brilliant blue platform for ultra-trace voltammetric analysis of rosuvastatin in tablets and human plasma†}

\begin{abstract}
Marwa R. El-Zahry (iD * and Marwa F. B. Ali
The enhancement effect of reduced graphene oxide ( $\mathrm{rGO}$ ) combined with silver nanocomposite supported on poly brilliant blue (PBB) platform was investigated for ultra-trace analysis of rosuvastatin (RS). Herein, in situ electrochemical deposition of the silver nanoparticles (AgNPs) and rGO hybrid was performed on the surface of the polymerized brilliant blue (PBB) platform. The developed (AgNPs-rGO/PBB) electrode showed an enhanced catalytic activity toward the oxidation of RS. The modified electrodes AgNPs-rGO/ PBB and AgNPs/PBB required an overpotential of 0.68 and $1.06 \mathrm{~V}$ to achieve a current density of $10 \mathrm{~mA}$ $\mathrm{cm}^{-2}$, and their corresponding Tafel slopes were calculated to be 70 and $105 \mathrm{mV} \mathrm{dec}{ }^{-1}$, respectively. Further, rGO and silver nanocomposites properties were characterized by scanning electron microscopy (SEM), cyclic voltammetry (CV), square wave voltammetry (SWV), linear sweep voltammetry (LSV), and electrochemical impedance spectroscopy (EIS). Additionally, the formation of GO and AgNPs-rGO was confirmed by Raman spectroscopy and Fourier transform infrared spectroscopy (FT-IR). Under the optimized conditions, the electrochemical sensor showed a remarkable response for quantitation of RS over a wide range of concentrations $5 \times 10^{-9}$ to $5 \times 10^{-7} \mathrm{~mol} \mathrm{~L}^{-1}(r=0.9988)$, with a limit of detection $2.17 \times 10^{-9} \mathrm{~mol} \mathrm{~L}{ }^{-1}$. The electrochemical performance of the studied electrode showed high reproducibility and suitability for tablets and human plasma.
\end{abstract}

\section{Introduction}

Recently, bi- and multi-layered modified electrodes have attracted much attention for the analysis of pharmaceutical and environmental samples due to their high sensitivity, selectivity and electro-catalytic activity. ${ }^{1}$

Graphene is a source of most graphitic materials and contains one single layer of carbon atoms. The most interesting properties of graphene are high mechanical strength and tunable optical properties. Among the various methods of graphene exfoliation, oxidation of graphite using strong oxidizing agents forming graphene oxide (GO), a non-conductive hydrophilic material, received great attention due to its simple synthetic protocol. Hummer's method was commonly used for the oxidation of graphite. ${ }^{2}$ To retain the aromatic backbone, GO was reduced either chemically using different reagents or electrochemically by applying more negative potential to reduce the

Pharmaceutical Analytical Chemistry Department, Faculty of Pharmacy, Assiut University, Assiut, 71526, Egypt. E-mail: marwazahry@aun.edu.eg; Tel: $+20-2411520$

$\dagger$ Electronic supplementary information (ESI) available. See DOI: $10.1039 / \mathrm{c} 8 \mathrm{ra10415h}$ oxygen functional group present on the surface of GO. ${ }^{3}$ It was reported that the electrochemically reduced graphene oxide will be more conducting than that prepared from chemical reduction. ${ }^{4}$

Incorporation of metal nanostructures with graphene or rGO greatly influences the electrochemical biosensors which accompanied by an improvement in biosensor sensitivity due to the synergistic effect between rGO and the metal nanostructures. In this study; silver nanoparticles (AgNPs) were selected due to their high conductivity, high surface reaction activity, high catalytic efficiency, strong adsorption ability, high spectral properties and ease of preparation.,

Brilliant blue (BB), is a triphenylmethane dye widely used for protein staining in analytical biochemistry field. BB was chosen as a combined valence compound applied on the electrode surface via electro-polymerization forming electro-active layers of poly brilliant blue (PBB). The well-organized layers of PBB over the surface of pencil graphite electrode (PGE) act as a platform for dispersion of the in situ deposited AgNPs-rGO hybrid nanocomposites.

Rosuvastatin (RS) is a highly effective anti-hyperlipidemic drug acts by inhibiting 3-hydroxy-3-methylglutaryl coenzyme A (HMG-CoA) reductase enzyme, which catalyzes the conversion 
of HMG-CoA to mevalonate in cholesterol biosynthesis. ${ }^{7}$ RS reduces low-density lipoprotein (LDL), inhibits hepatic synthesis of very-low-density lipoprotein (VLDL) and lowers triglycerides levels in hypertriglyceridemic patients. ${ }^{8} \mathrm{RS}$ has shown a great effect to reduce the rate of mortality in coronary patients and prevent cardiovascular events in ischemic heart disease patients. Owing to its importance, several techniques were reported for determination of RS such spectrophotometry, ${ }^{9-12}$ spectrofluorimetry, ${ }^{\mathbf{1 3}}$ capillary electrophoresis, ${ }^{\mathbf{1 4}}$ HPTLC, ${ }^{15-17}$ electrochemistry, ${ }^{18-23}$ and HPLC. ${ }^{24-29}$ Electrochemical techniques exhibited a recent interest owing to its high sensitivity, good selectivity, reproducibility, and rapidity when compared with other analytical methods. Hence, the presented article selected square wave voltammetric (SWV) technique as the technique of choice for detection of RS in different matrices.

Moreover, PGE was selected in the present investigation because of its high electrochemical reactivity, low background current, good mechanical rigidity, disposability, low cost and commercial availability. It offers renewal surface which is simpler and faster than polishing procedures, ease in modification and more environmentally friendly. ${ }^{30,31}$

In the present work, an electrochemical sensing platform based on in situ electro-deposition of AgNPs-rGO (outer layer) on PBB surface (inner layer) was fabricated to modify a graphite electrode (PGE). The presented modified electrode showed an excellent sensitivity compared with other reported electrochemical and HPLC methods. ${ }^{18-29}$

The main aim of this study is to investigate the role of rGO in facilitating the charge transfer between the AgNPs and the polymerized BB on PGE surface. The modified electrode was characterized by cyclic voltammetry (CV), square wave voltammetry (SWV), linear sweep voltammetry (LSV), electrochemical impedance spectroscopy (EIS). Scanning electron microscope (SEM) was used to follow the morphological properties of the electrode after each preparation step. The formation of GO and AgNPs-rGO was confirmed using Raman and FT-IR spectroscopy. The proposed SWV method was optimized, validated and successfully employed for determination of RS in pharmaceutical tablet and human plasma following ICH guidelines. ${ }^{32}$

\section{Experimental}

All chemicals, instruments and the preparation steps were mentioned in the ESI. $\dagger$ The fabrication process of the newly developed electrode was shown in Scheme 1.

\section{Results and discussion}

\section{Characterization of AgNPs-rGO/PBB modified electrode}

FT-IR and Raman spectroscopy characterization of AgNPsrGO hybrid nanocomposite. GO was prepared following the modified Hummer's procedure, ${ }^{2}$ the synthesized GO powder was characterized using FT-IR and Raman spectroscopic techniques. As shown in Fig. S1A (shown in ESI†), the FT-IR spectrum of graphite exhibited no significant peak indicating the chemical inertness of graphite, while GO spectrum showed

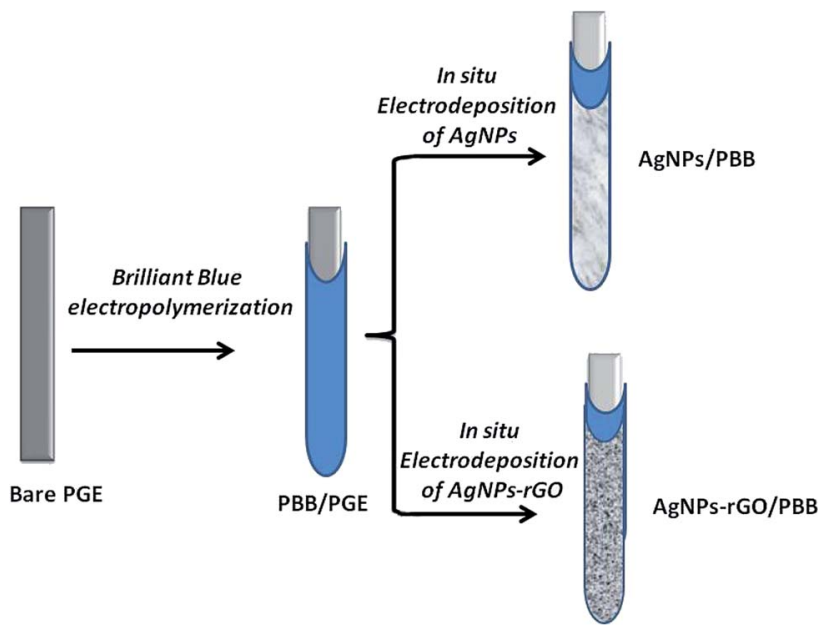

Scheme 1 Schematic presentation of the steps involved in fabrication of AgNPs-rGO/PBB and AgNPs/PBB bi-layered modified electrodes.

various functional groups. An intense peak at $3430 \mathrm{~cm}^{-1}$ is attributed to $\mathrm{O}-\mathrm{H}$ stretching vibration indicating the presence of $\mathrm{OH}$ or $\mathrm{COOH}$ groups in GO structure. The peak at $1732 \mathrm{~cm}^{-1}$ is due to stretching vibration of carbonyl group $(\mathrm{C}=\mathrm{O})$, while $\mathrm{O}-\mathrm{H}$ deformation is observed at $1410 \mathrm{~cm}^{-1}$ and $\mathrm{C}-\mathrm{O}$ stretching vibration of alkoxy group $\left(1050 \mathrm{~cm}^{-1}\right)$ is also observed. ${ }^{33}$

In situ deposition of AgNPs-rGO on the electrode surface was achieved electrochemically via reduction of oxygen groups present on the surface of GO. The fabricated AgNPs-rGO electrode was further characterized by monitoring Raman spectra of both GO and AgNPs-rGO. Fig. S1B $\uparrow$ shows the recorded Raman spectrum of GO which displays two main peaks at 1349 and $1595 \mathrm{~cm}^{-1}$ corresponding to $\mathrm{D}$ and $\mathrm{G}$ bands, respectively. ${ }^{31}$ Comparing with GO, the Raman spectrum of AgNPs-rGO was recorded in which $\mathrm{D}$ and $\mathrm{G}$ bands were shifted to lower wavenumbers at 1347 and $1591 \mathrm{~cm}^{-1}$ which is attributed to the reduction of GO during the electrodeposition process. Additionally, the Raman spectrum of AgNPs-rGO showed a greater intensity compared to GO, which confirms the incorporation of AgNPs to rGO nanocomposite and the surface enhanced Raman scattering (SERS) produced by AgNPs. ${ }^{34}$ The electrochemical deposition was carried out using cyclic voltammetric technique, where different factors such as the GO concentration and deposition potential were optimized. The maximum current peak of RS was obtained using $0.25 \mathrm{mg} \mathrm{mL}^{-1} \mathrm{GO}$ using potential ranged from $0 \mathrm{~V}$ to $-1.5 \mathrm{~V}$ at $100 \mathrm{mV} \mathrm{s}^{-1}$ scan rate.

Scanning electron microscopy (SEM) characterization. In order to follow the morphological changes that appear on the surface of PGE during the preparation procedures, SEM images of bare PGE, PBB layer and AgNPs-rGO/PBB surfaces were collected. Fig. 1A shows the characteristic features of bare PGE surface with wrinkling and overlapped sheets. After polymerization of BB monomers on PGE surface (Fig. 1B); a rough and porous surface is clearly seen with the formed flocculated structures indicating the formation of PBB platform. In Fig. 1C, there are bright particles formed after the in situ electrodeposition of AgNPs-rGO on PBB platform which provides a good dispersion of AgNPs all over the surface of the electrode. 

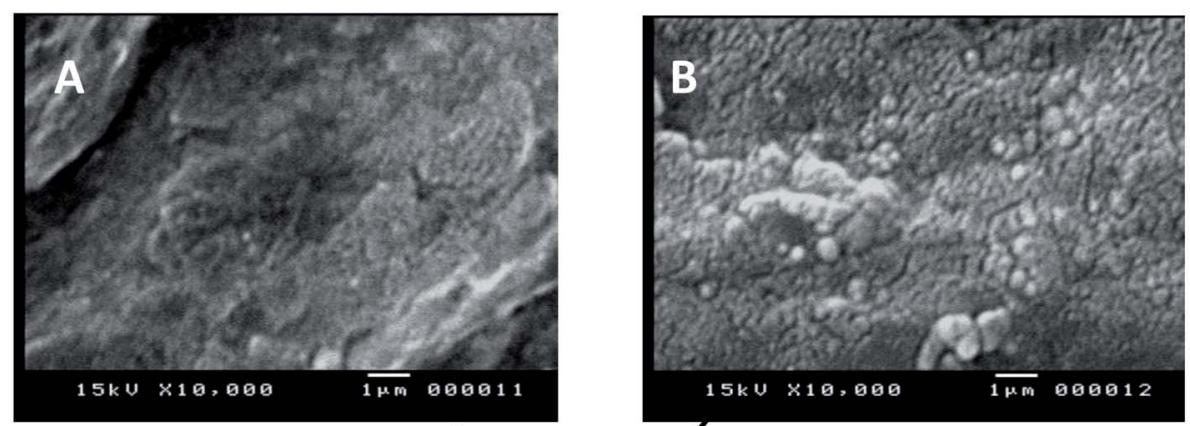

Bare PGE

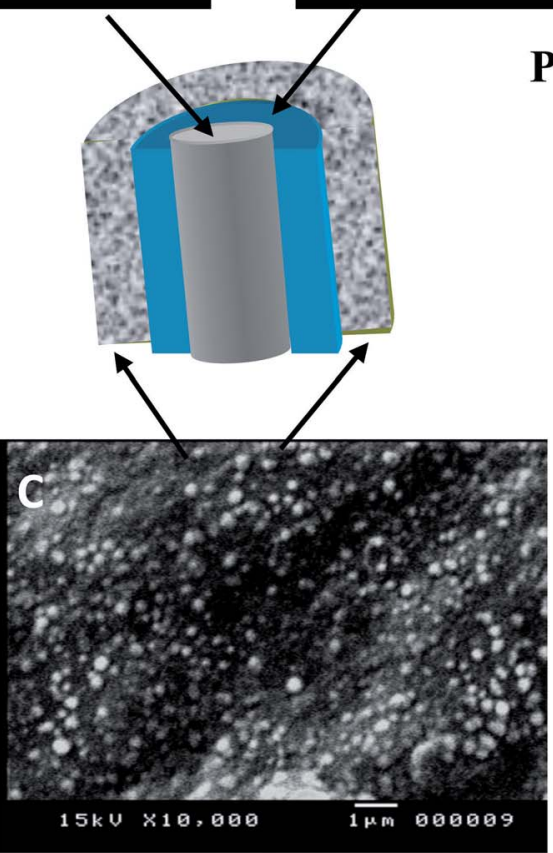

PBB/PEG

\section{AgNPs-rGO/PBB}

Fig. 1 Schematic diagram of the bi-layered modified electrode accompanied with SEM images of the boundaries surface of (A) bare PGE, (B) PBB/PGE and (C) AgNPs-rGO/PBB.

Electrochemical behavior of AgNPs-rGO/PBB toward RS electro-oxidation. To demonstrate the electrochemical performance of the modified electrodes, cyclic voltammograms of RS were scanned using bare PGE, rGO/PBB and AgNPs-rGO/PBB electrodes (Fig. 2A). Comparing with $\mathrm{rGO} / \mathrm{PBB}$ and bare PGE, the enhancement effect of AgNPs-rGO/PBB modified electrode on the oxidation current peak of $3.5 \times 10^{-7} \mathrm{~mol} \mathrm{~L}^{-1} \mathrm{RS}$ was observed at $1.15 \mathrm{~V}(v s . \mathrm{Ag} / \mathrm{AgCl})$. Additionally, $\mathrm{SWV}$ technique was utilized to investigate the electrocatalytic oxidation behavior of RS using bare PGE and glassy carbon electrode (GCE).

It can be revealed that $\mathrm{rGO}$ enhances the electroactive properties of the studied electrode; this behavior may be attributed to the synergistic effect of rGO incorporated with silver nanocomposites. The improved performance is based on facilitating the electron transfer between the outer layer AgNPsrGO and the inner PBB layers through the conducting material rGO. Additionally, increase the surface active area of the studied electrode caused by AgNPs incorporation could be another reason for the improved electroactive properties. ${ }^{35}$
In order to demonstrate rGO significance on the electrode performance, an overlay of another modification, AgNPs/PBB electrode without using rGO, was constructed and compared electrochemically with AgNPs-rGO/PBB toward electrooxidation of RS (Fig. 2B). The AgNPs/PBB exhibited a lower activity towards RS oxidation. This decline in the electrical response confirms the significance of rGO as a conducting element providing an excellent charge transfer between the presented layers. ${ }^{36}$

Optimization of the inner poly brilliant blue layer (PBB). As the thickness of the polymer layer affects the electro-catalytic activity of the modified electrode, the optimal conditions of $\mathrm{BB}$ polymer growth on the modified electrode were investigated. From the obtained results (Fig. $3 \mathrm{~A}-\mathrm{C}$ ), it was found that the optimum potential range of the $\mathrm{BB}$ electro-polymerization process is $-0.5 \mathrm{~V}$ to $1.5 \mathrm{~V}$ using $100 \mathrm{mV} \mathrm{s}^{-1}$ scan rate for 7 multiple cycles.

Effect of scan rate and the suggested electro-oxidation mechanism of RS. The effect of scan rate ranged from $0.05 \mathrm{~V}$ to $1.0 \mathrm{~V} \mathrm{~s}^{-1}$ was investigated at AgNPs-rGO/PBB modified 

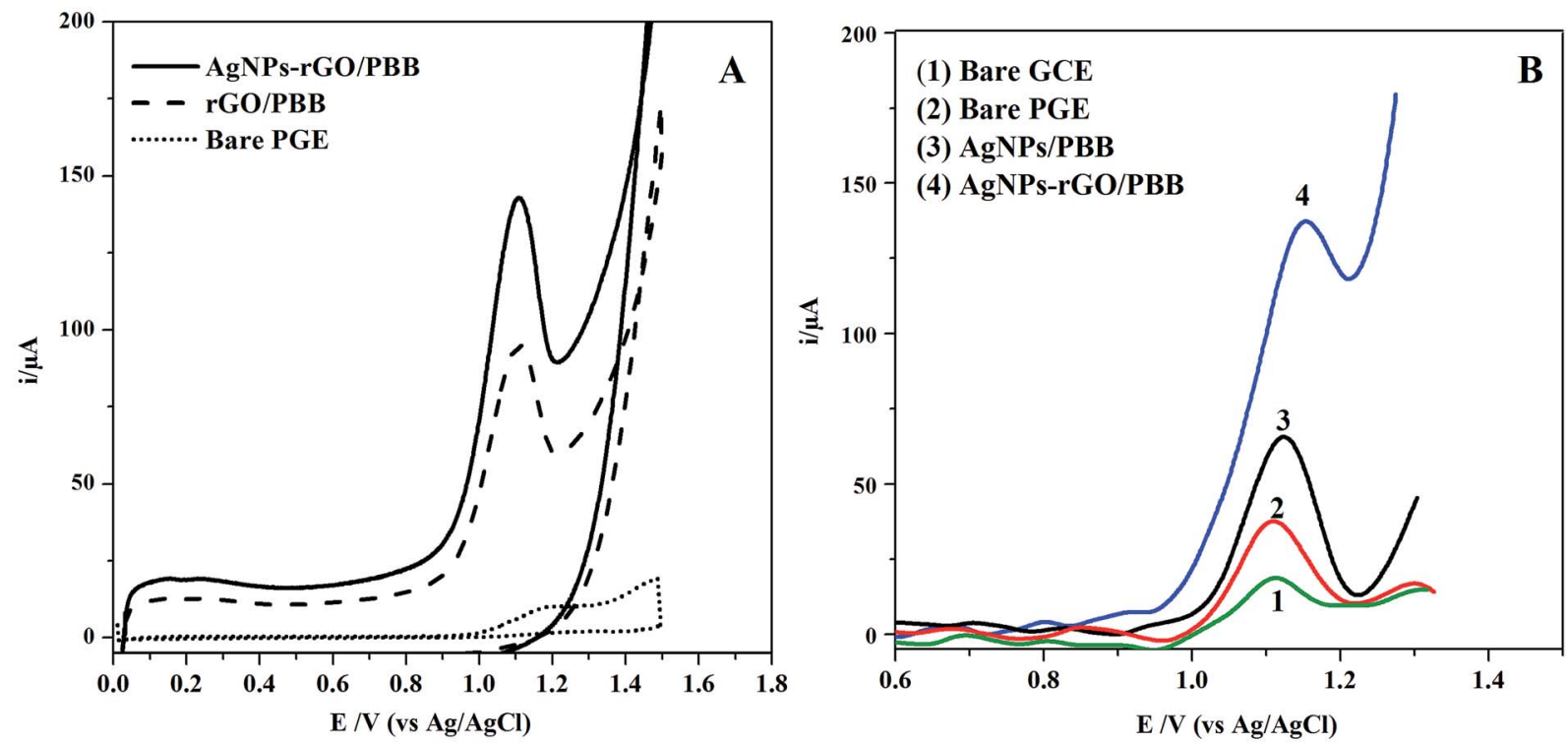

Fig. 2 (A) CV curves of RS recorded at bare PGE, rGO/PBB and AgNPs-rGO/PBB in $0.15 \mathrm{~mol} \mathrm{~L}^{-1} \mathrm{H}_{2} \mathrm{SO}_{4}$ under the optimum conditions and (B) SWV curves of $3.5 \times 10^{-7} \mathrm{~mol} \mathrm{~L}^{-1}$ of RS at different electrodes.
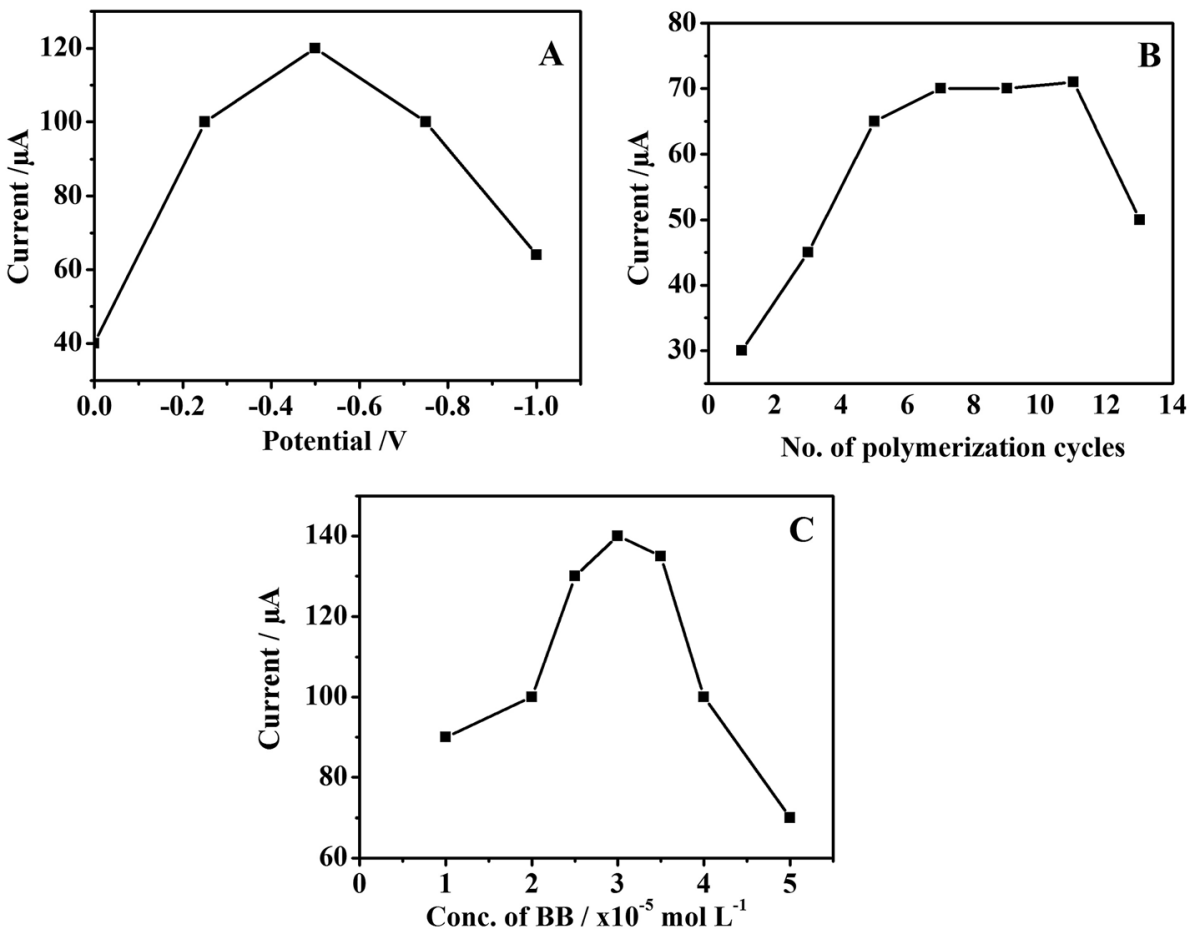

Fig. 3 Effect of electro-polymerization parameters (A) initial potential (B) number of polymerization cycles and (C) concentration of BB on the current intensity of $3.5 \times 10^{-7} \mathrm{~mol} \mathrm{~L}^{-1} \mathrm{RS}$ recorded at the modified electrode.

electrode. According to Randles-Ševciiks equation, the peak current is directly proportional to the scan rate. ${ }^{37}$ Fig. 4 shows an increase in the anodic peak current accompanied with a shift in the anodic peak potential to more positive values with increasing the scan rate, which is observed when the oxidation reaction is irreversible. A good linearity was obtained upon plotting the anodic peak current against the studied range of scan rate following the equation below:

$$
I_{\mathrm{p}}(\mu \mathrm{A})=105.5+31.5 \nu,(R=0.9972)
$$

Fig. 5 describes the linear plot of $\log I_{\mathrm{p}}$ versus logarithm of scan rate $(\log \nu)$ which can be described by the following regression equation:

$$
\log I_{\mathrm{p}}(\mu \mathrm{A})=2.09+0.473 \log \nu,(R=0.9949)
$$




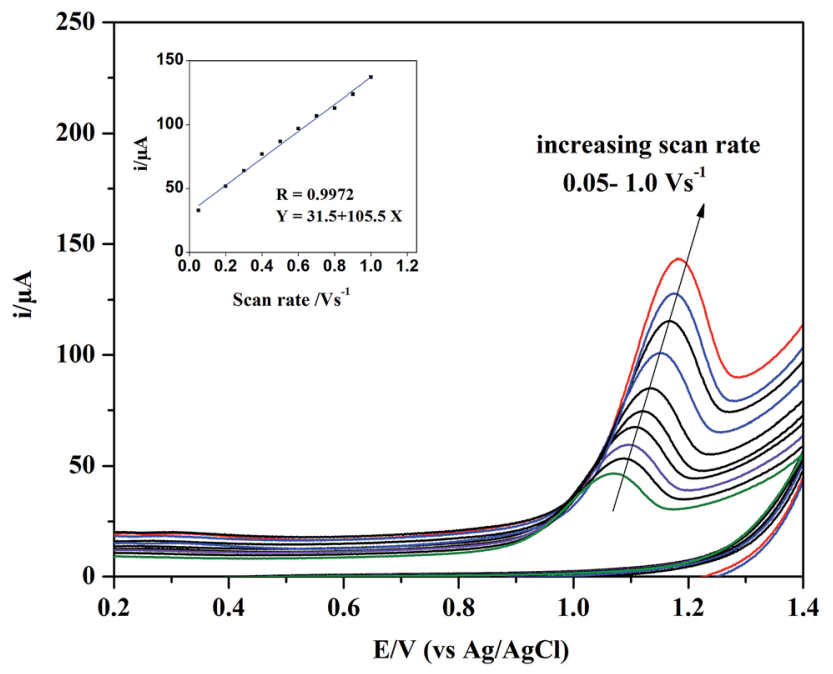

Fig. 4 The effect of different scan rates on CV curve of $3.5 \times$ $10^{-7} \mathrm{~mol} \mathrm{~L}^{-1} \mathrm{RS}$. Inset: dependence of oxidation peak current $\left(I_{\mathrm{p}} / \mu \mathrm{A}\right)$ of $\mathrm{RS}$ on scan rate $\left(\mathrm{V} \mathrm{s}^{-1}\right)$.

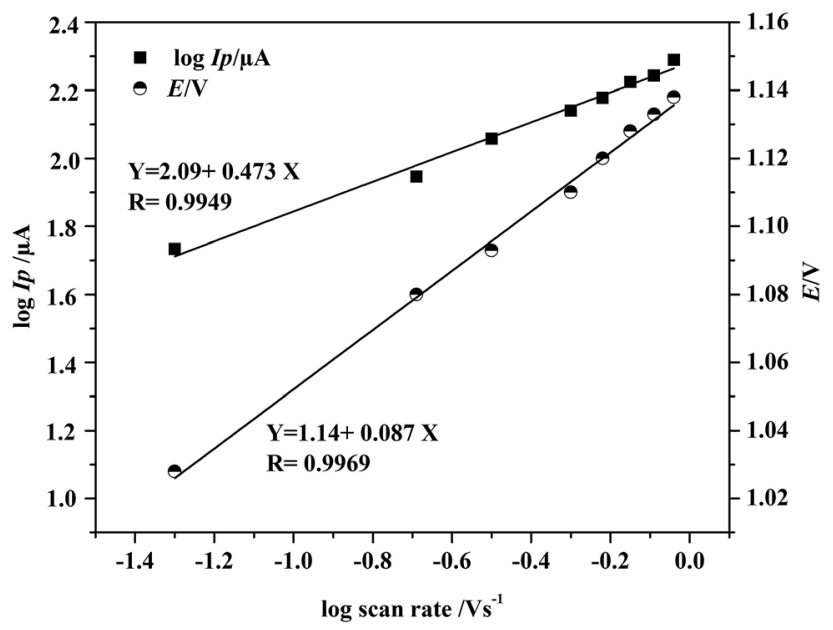

Fig. 5 Dependence of the logarithm of peak current $(\log / \mathrm{p} / \mu \mathrm{A})$ and the oxidation peak potential $(E / V)$ on logarithm of scan rate $\left(V s^{-1}\right)$.

The slope of the above linear equation was calculated to be 0.47 , a result close to the theoretical value of 0.5 predicted by Randles-Ševćiks equation for the diffusion-controlled process. Additionally, the relationship between the peak potential $E$ and logarithm of scan rate $(\log \nu)$ was plotted and provided a linear regression equation as follow:

$$
E(\mathrm{~V})=1.14+0.087 \log \nu,(R=0.9969)
$$

Based on Laviron equation, ${ }^{38}$ a relationship between the potential and the scan rate, the number of electron $(n)$ transferred in the rate limiting step can be calculated as given by the following equation:

Slope of the plot E versus $\log \nu=2.2303 R T / \alpha n F$
$R$ is the universal gas constant $\left(8.314 \mathrm{~J} \mathrm{~mol}^{-1} \mathrm{~K}^{-1}\right)$, taking $T$ with $298 \mathrm{~K}, \alpha$ is the transfer coefficient $(=0.5$ in totally irreversible reactions) and $F$ is the Faraday constant $\left(96.480 \mathrm{C} \mathrm{mol}^{-1}\right)$. After substitution the slope with 0.087 , the value of $\alpha n$ will be equal to 0.62 and the number of electron is estimated to be $1.2(\approx 1.0$ electron) which is in agreement with the previously reported researches discussing electro-oxidation of $\mathrm{RS}^{\mathbf{1 9 - 2 1}}$ As it previously reported, the electro-oxidation process of RS is based on Kolbe electrolysis reaction of the carboxylic acid group. This reaction is based on decarboxylation dimerization of two carboxylate ions. The protonated carboxyl group loses one electron and one proton in an irreversible oxidation reaction with the formation of a carboxyl radical. Due to the instability of this radical, it rapidly loses carbon dioxide $\left(\mathrm{CO}_{2}\right)$, producing a Ccentered radical. Finally, the C-centered radicals suffer a coupling to produce the dimer. ${ }^{39}$ The scheme as reported for the RS oxidation reaction is shown in ESI (Fig. S2 $\dagger$ ).

Electrochemical characterization of the modified electrode using potassium ferricyanide. The electrochemical performance of the bare and modified PGE electrodes were evaluated using the redox activity of $1.0 \mathrm{mmol} \mathrm{L}{ }^{-1}$ potassium ferricyanide $\mathrm{K}_{3}\left[\mathrm{Fe}(\mathrm{CN})_{6}\right]$ in $0.5 \mathrm{~mol} \mathrm{~L}^{-1}$ of $\mathrm{KCl}$ using $\mathrm{CV}$ technique (Fig. $6 \mathrm{~A}$ ). The enhanced performance of AgNPs-rGO/PBB modified electrode was clearly shown in terms of higher peak current intensities of the reduction-oxidation peak of potassium ferricyanide redox system. This behavior was further confirmed by carrying out electrochemical impedance spectroscopy (EIS) experiments that showed lower series resistance for each of the modified electrodes than the bare one.

The interfacial properties of the modified electrodes were studied using EIS method. Fig. 6B represents the Nyquist plots of the three different studied electrodes, namely (1) bare PGE, (2) AgNPs/PBB and (3) AgNPs-rGO/PBB. It was performed at a potential amplitude of $10 \mathrm{mV}$ at frequency range of $1.0 \mathrm{~Hz}$ and $10 \mathrm{KHz} . R_{\mathrm{ct}}$ is the diameter of the semi-depressed circle and corresponds to charge transfer process across PGE electrode, $W_{\mathrm{S}}$ is the generalized Warburg impedance for finite diffusion of the reactants. The Nyquist plot describes the charge transfer resistance $\left(R_{\mathrm{ct}}\right)$ and its relationship with the charge transfer across the electrode surface. $R_{\mathrm{ct}}$ values were calculated using Zview software (Z-view version $3.5 \mathrm{~d}$ ). The diameter of the semidepressed circle is the smallest in case of AgNPs-rGO/PBB. The small values of $R_{\mathrm{ct}}$ indicate the enhancement of the electrical conductivity and the speed of the electron transfer. The Nyquist plots of the modified electrodes show linear curves indicating a diffusion behavior comparing with the bare electrode which shows a clear circular curve. Fig. 6C represents the change in charge transfer resistance of potassium ferricyanide redox system as a result of different treatment on the PGE electrode.

The overpotential $(\eta)$ required at the current density (mA $\mathrm{cm}^{-2}$ ) is considered a benchmark to evaluate the electrocatalytic activity of the proposed electrode. ${ }^{40}$ As shown in Fig. 7A, the modified electrode AgNPs-rGO/PBB achieved a lower overpotential $\sim 0.68 \mathrm{~V}$ to reach $10 \mathrm{~mA} \mathrm{~cm}{ }^{-2}$ current density as compared to AgNPs/PBB $(\sim 1.06 \mathrm{~V})$. The lower overpotential value of AgNPs-rGO/PBB contributes to the higher 

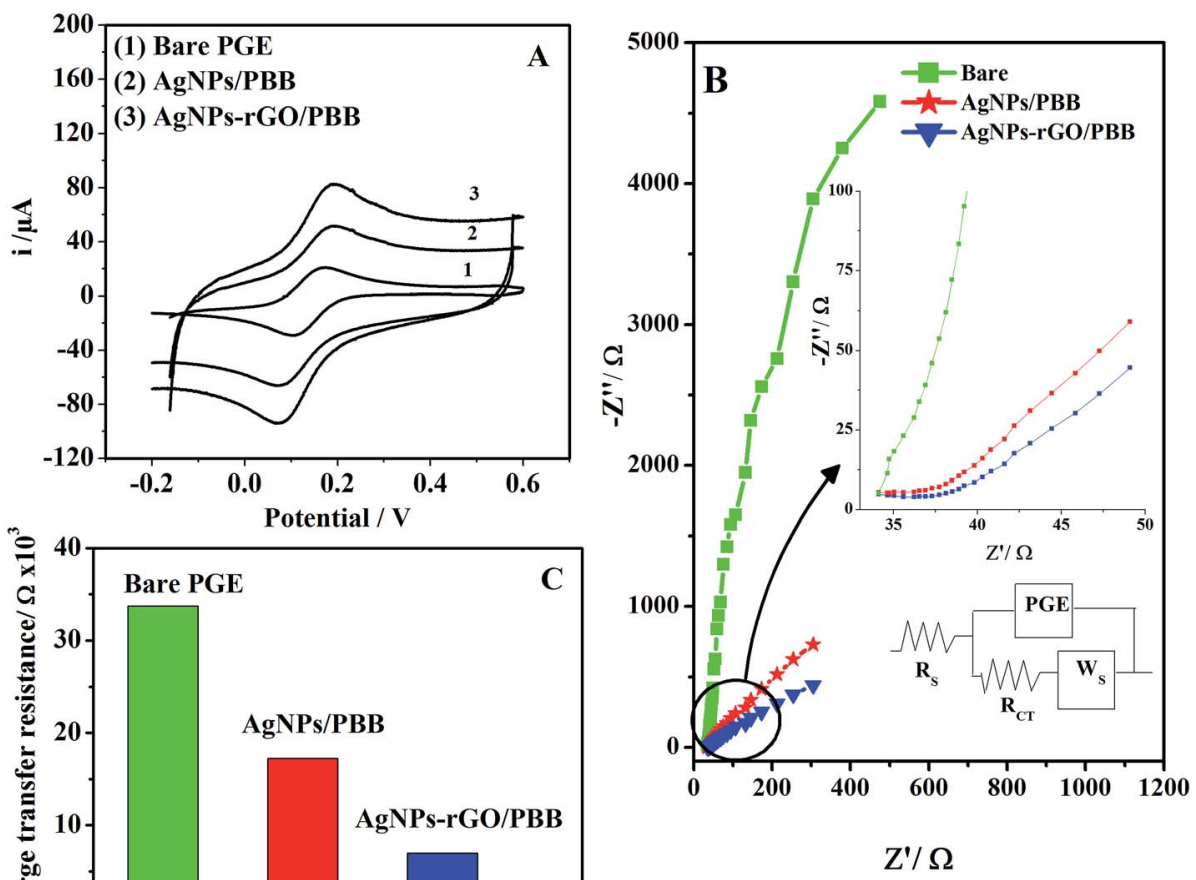

Fig. 6 (A) CV curves, (B) Nyquist plots of $1.0 \mathrm{mmol} \mathrm{L}^{-1}$ potassium ferricyanide in $0.5 \mathrm{~mol} \mathrm{~L} \mathrm{~K}^{-1} \mathrm{KCl}$ monitored on (1) bare PGE, (2) AgNPs/PBB and (3) AgNPs-rGO/PBB electrode. Inset: enlarged view at lower frequency range, and (C) the corresponding histogram of change in charge transfer resistance. Working conditions: scan rate $100 \mathrm{mV} \mathrm{s}^{-1}$ and potential range $-0.2 \mathrm{~V}$ to $0.6 \mathrm{~V}$.

electrocatalytic activity. For better understanding the catalytic kinetics of the studied electrodes, the Tafel plots $(\log j v s . \eta)$ were constructed for the studied electrodes by monitoring linear scan voltammetry (LSV) (Fig. 7B) at scan rate $10 \mathrm{~V} \mathrm{~s}^{-1}$ and fitted into the Tafel equation,

$$
\eta=a+b \log j
$$

where $\eta$ is overpotential and $j$ is the current density. The fitted Tafel plots of the bare PGE and the modified electrodes are presented in Fig. 7C. AgNPs-rGO/PBB shows Tafel slope of $70 \mathrm{mV} \mathrm{dec}{ }^{-1}$ lower than that of AgNPs/PBB $\left(105 \mathrm{mV} \mathrm{dec}{ }^{-1}\right)$ suggesting that AgNPs-rGO/PBB exhibits the best electrocatalytic behavior over the other studied electrodes.

According to Randles-Sevcik equation, the surface active areas of the studied electrodes have been calculated after each modification step:

$$
I_{\mathrm{pa}}=\left(2.69 \times 10^{5}\right) n^{3 / 2} A_{\mathrm{eff}} D_{\mathrm{R}}{ }^{1 / 2} C_{0} \nu^{1 / 2}
$$

where $n$ is the number of electron transfer, $A_{\text {eff }}$ is the surface area of the electrode $\left(\mathrm{cm}^{2}\right), D_{\mathrm{R}}$ is the diffusion coefficient $\left(\mathrm{cm}^{2}\right.$ $\left.\mathrm{s}^{-1}\right), \nu$ is the scan rate $\left(\mathrm{V} \mathrm{s}^{-1}\right)$ and $C_{0}$ is the concentration of potassium ferricyanide $\left(\mathrm{mol} \mathrm{cm}{ }^{-2}\right)$. The surface active areas of bare, AgNPs/PBB and AgNPs-rGO/PBB were calculated to be: 44 , 86 and $182.2 \mathrm{~mm}^{2}$, respectively. The results in Fig. 7D indicate the enhancement effect of the incorporated rGO layer with AgNPs supported on PBB platform causing a cumulative increase in the surface active area and hence in the oxidation peak current of RS on the fabricated electrode.
Effect of supporting electrolyte $\mathrm{pH}$ and voltammetric parameters. In order to develop a highly sensitive and selective response of the modified electrode, several parameters such as $\mathrm{pH}$ of supporting electrolyte and various instrumental parameters that affect the electro-oxidation of RS have been optimized. The $\mathrm{pH}$ of the supporting electrolyte has a significant effect on the electro-oxidation of RS at the modified electrode. The electro-oxidation of RS $\left(2.5 \times 10^{-7} \mathrm{~mol} \mathrm{~L}^{-1}\right)$ was investigated over $\mathrm{pH}$ range $2.5-8.0$ in different electrolytes. It was found that oxidation peak current increases with decreasing $\mathrm{pH}$ value until it reaches $\mathrm{pH}$ 2.5. Therefore, different acid solutions such as sulphuric, hydrochloric, acetic and phosphoric acid were tested, and sulphuric acid showed the highest results. Further, different concentrations of sulphuric acid over the range $0.05-0.25 \mathrm{~mol} \mathrm{~L}^{-1}$ were tried. Sulphuric acid of $0.15 \mathrm{~mol} \mathrm{~L}^{-1}$ was selected as the optimum concentration for RS determination which well agreed with the previously reported method. ${ }^{21}$ In addition, different SWV parameters were optimized, including; pulse height, step height, frequency, initial potential and deposition time. Table 1 summarized the investigated parameters and the obtained results that give the maximum response after optimization.

\section{Validation of the proposed method}

Linearity and detection limit. Under the optimum conditions; SWV method was utilized for construction of RS calibration plot over the concentration range of $5 \times 10^{-9}$ to $5 \times$ $10^{-7} \mathrm{~mol} \mathrm{~L}^{-1}$ using a scan rate of $40 \mathrm{mV} \mathrm{s}^{-1}$ (Fig. 8). The limit of detection $\left(\mathrm{LOD}=3 S_{\mathrm{b}} / \mathrm{m}\right)$ and the limit of quantitation $(\mathrm{LOQ}=$ 

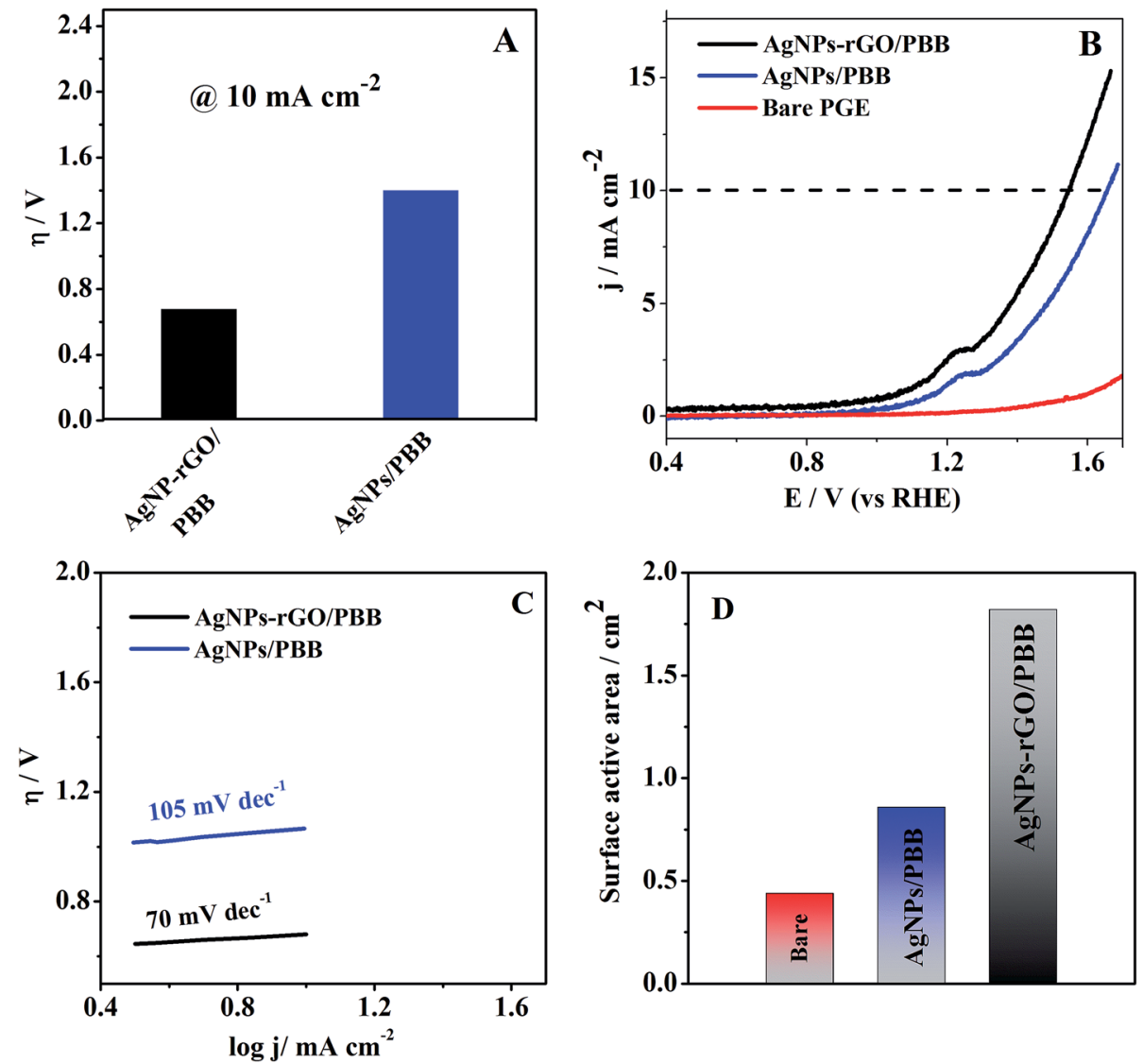

Fig. 7 (A) Histograms of the overpotential required for $10 \mathrm{~mA} \mathrm{~cm}{ }^{-2}$ current density, (B) LSV polarization curves monitored at low scan rate (10 mV $\mathrm{s}^{-1}$ ) showing the catalytic activity of the studied bare and modified electrodes, (C) Tafel plots of AgNPs-rGO/PBB, and AgNPs/PBB and (D) histograms of the surface active areas of the studied electrodes.

Table 1 Optimization of SWV parameters for the determination of RS using AgNPs-rGO/PBB modified electrode

\begin{tabular}{lll}
\hline Parameters & Studied range & Best response \\
\hline Pulse height $(\mathrm{mV})$ & 3 to 30 & 15 \\
Step height $(\mathrm{mV})$ & 1 to 30 & 10 \\
Frequency $(\mathrm{Hz})$ & 50 to 250 & 200 \\
Initial potential $(\mathrm{V})$ & -0.6 to 0.6 & 0.0 \\
Deposition time $(\mathrm{s})$ & 10 to 180 & 30
\end{tabular}

$\left.10 S_{\mathrm{b}} / m\right)$; where $S_{\mathrm{b}}$ is the standard deviation of the intercept and $m$ is the slope of the calibration line are equal to $2.17 \times 10^{-9}$ and $6.66 \times 10^{-9} \mathrm{~mol} \mathrm{~L}^{-1}$, respectively. All the linear regression parameters of the proposed method are presented in Table 2.

Precision. To investigate the stability of the AgNPs-rGO/PBB performance, the precision of the proposed method was studied through inter- and intra-day repeatability measurements. Intraday precision was evaluated by repeating the analysis of three different concentration levels of RS working solution. This study was repeated over a period of three working days to study the inter-day repeatability. The intra- and inter-day precision were expressed as relative standard deviation (\% RSD) which was found to be less than $3.96 \%$, indicating high stability, good repeatability and reproducibility of the modified electrode (Table 2).
Interference study. In order to estimate the selectivity of the modified electrode for determination of RS, the effect of different interfering compounds have been investigated. Hence, $2.5 \times$ $10^{-7} \mathrm{~mol} \mathrm{~L}^{-1}$ of RS solution was determined using the proposed

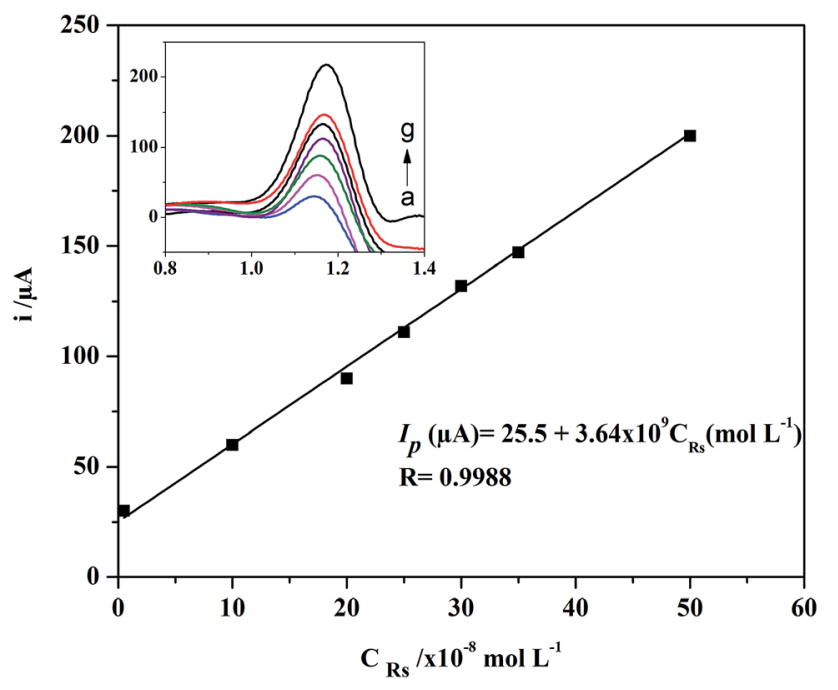

Fig. 8 Linear plot between the oxidation peak current and different concentrations of RS. Inset: SWV curves of RS monitored on the studied modified AgNPs-rGO/PBB electrode. 
Table 2 Statistical parameters and precision values of the proposed SWV method using AgNPs-rGO/PBB modified electrode

\begin{tabular}{|c|c|}
\hline Parameters & The calculated value \\
\hline Linearity range $\left(\mathrm{mol} \mathrm{L}^{-1}\right)$ & $5 \times 10^{-9}$ to $5 \times 10^{-7}$ \\
\hline Intercept $(\mu \mathrm{A}) \pm \mathrm{SD}^{a}$ & $25.5 \pm 2.34$ \\
\hline Slope $\left(\mu \mathrm{A}\left(\mathrm{mol} \mathrm{L}^{-1}\right)^{-1}\right) \pm \mathrm{SD}^{b}$ & $3.64 \times 10^{9} \pm 9.21 \times 10^{-7}$ \\
\hline $\mathrm{SSE}^{c}$ & $50.1 \times 10^{-8}$ \\
\hline Correlation coefficient $(R)$ & 0.9988 \\
\hline $\mathrm{LOD}^{d}\left(\mathrm{~mol} \mathrm{~L}^{-1}\right)$ & $2.17 \times 10^{-9}$ \\
\hline $\mathrm{LOQ}^{e}\left(\mathrm{~mol} \mathrm{~L}^{-1}\right)$ & $6.66 \times 10^{-9}$ \\
\hline Intra-day precision ${ }^{f}\left(\% \mathrm{RSD}, n=3^{g}\right.$ ) & $\leq 3.45$ \\
\hline Inter-day precision ${ }^{f}\left(\% \mathrm{RSD}, n=3^{g}\right.$ ) & $\leq 3.96$ \\
\hline
\end{tabular}

Table 3 Interference study using different concomitant substances found in the pharmaceutical formulations of RS

\begin{tabular}{llc}
\hline Concomitant substances & Ratio, RS : concomitant & Recovery $\% \pm \mathrm{SD}^{a}$ \\
\hline Ascorbic acid & $1: 10$ & $98.6 \pm 0.56$ \\
& $1: 100$ & $97.7 \pm 0.77$ \\
Citric acid & $1: 10$ & $98.4 \pm 0.82$ \\
& $1: 100$ & $96.7 \pm 0.98$ \\
Starch & $1: 10$ & $99.6 \pm 1.34$ \\
Magnesium stearate & $1: 100$ & $97.7 \pm 0.9$ \\
& $1: 10$ & $100.6 \pm 0.66$ \\
Uric acid & $1: 100$ & $99.7 \pm 0.77$ \\
& $1: 10$ & $99.1 \pm 1.32$ \\
Glucose & $1: 100$ & $97.4 \pm 0.45$ \\
& $1: 10$ & $99.9 \pm 0.33$ \\
Sucrose & $1: 100$ & $97.0 \pm 0.18$ \\
& $1: 10$ & $99.4 \pm 0.72$ \\
a Average of three determinations. & $99.1 \pm 0.23$
\end{tabular}

${ }^{a}$ Average of three determinations. modified electrode in the absence and presence of the following substances: ascorbic acid, citric acid, starch, glucose, sucrose, uric acid and magnesium stearate. As presented in Table 3, the ratio of $\mathrm{RS} /$ concomitant has been adopted to be $1: 10$ and $1: 100$. The adopted ratio $1: 100$ is much higher than that found in RS pharmaceutical dosage forms, as these substances are used in pharmaceutical formulations at a mass concentration of $2-5 \%$ $\mathrm{m} / \mathrm{m}$. From the presented results in Table 3, it can be inferred that there is no significant interference from these matrices on the voltammetric determination of RS.

Determination of RS in pharmaceutical tablets and human plasma. In order to demonstrate the applicability of the modified electrode as a sensing probe of RS in complicated matrices, RS concentrations were monitored in two different matrices; pharmaceutical dosage forms and human plasma. Initially, three pharmaceutical tablets with different RS contents were purchased from local market and analyzed using the modified electrode. The accuracy was studied and good recoveries were obtained (96.6-99.5\%) with good agreement with the claimed amounts as shown in Table 4 . The obtained results were statistically compared to those obtained by another reported method of RS determination. ${ }^{12}$ The calculated $t$ and $F$ values at 95\% confidence level were less than the tabulated ones indicating the high accuracy of the developed voltammetric method.

The recovery of RS from human plasma samples was measured by spiking the plasma sample with known amount of RS standard solution at four different concentration levels. Table 4 shows the found amounts and the calculated recovery percentages of RS quantitation in human plasma samples after simple liquid-liquid extraction method. The obtained recoveries, ranged from 97.2 to $101.2 \%$, indicate the analytical utility and the potentiality of the modified electrode to be used as an ultra-sensor for voltammetric determination of RS with excellent sensitivity and reproducibility.

Comparative study of the proposed and the previously reported methods. The sensitivity of the proposed method was compared with different reported methods for determination of RS

Table 4 Determination of RS in commercial pharmaceutical tablets and spiked human plasma using AgNPs-rGO/PBB modified electrode ${ }^{a}$

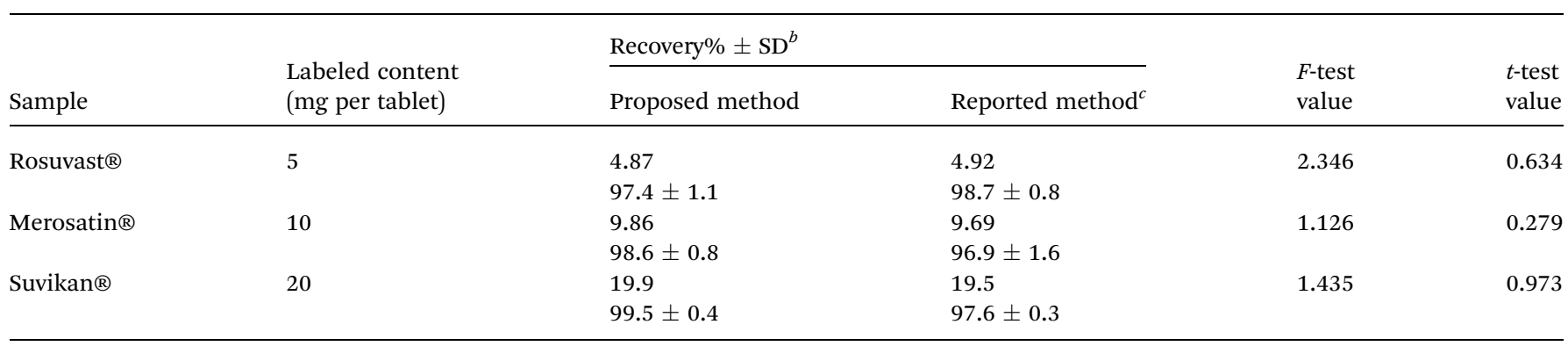

\begin{tabular}{llll}
\hline Sample & Added amount $\left(\mathrm{mol} \mathrm{L}^{-1}\right)$ & Found amount $\left(\mathrm{mol} \mathrm{L}^{-1}\right)$ & $\mathrm{Recovery}^{2} \pm \mathrm{SD}^{b}$ \\
\hline Human plasma & $1 \times 10^{-8}$ & $0.99 \times 10^{-8}$ & $99.5 \pm 1.0$ \\
& $10 \times 10^{-8}$ & $10.1 \times 10^{-8}$ & $101.2 \pm 0.3$ \\
& $20 \times 10^{-8}$ & $19.7 \times 10^{-8}$ & $98.6 \pm 1.0$ \\
& $40 \times 10^{-8}$ & $38.9 \times 10^{-8}$ & $97.2 \pm 0.5$
\end{tabular}

${ }^{a}$ Theoretical values at $95 \%$ confidence limit, $t=2.776$ and $F=19 .{ }^{b}$ Average of three determinations \pm SD. ${ }^{c}$ Ref. 12 . 
Table 5 Comparison between the proposed SWV method and the previously reported methods

\begin{tabular}{lll}
\hline Analytical method & LOD (mol L $)^{-1}$ & Ref. \\
\hline Proposed voltammetric method using AgNPs-rGO/PBB & $2.17 \times 10^{-9}$ & This method \\
Capillary electrophoresis & $1.0 \times 10^{-6}$ & $11 \times 10^{-9} /$ band \\
HPTLC & $0.3 \times 10^{-7}$ & 17 \\
Electrochemical method using glassy carbon electrode (GCE) & $0.4 \times 10^{-7}$ & 18 \\
HPLC/DAD & $0.3 \times 10^{-7}$ \\
UPLC/DAD & $6.0 \times 10^{-8}$ & 18 \\
Voltammetric using carbon nanotubes and graphene oxide & & 18 \\
electrode & $1.04 \times 10^{-6}$ \\
Electrochemical using a boron-doped diamond electrode (BDD) & $0.01 \times 10^{-6}$ \\
Electrochemical using HMDE & $1.2 \times 10^{-6}$ \\
Electrochemical using static mercury drop electrode (SMDE) & $0.1 \times 10^{-6}$ & 19 \\
HPLC/FL & $0.1 \times 10^{-6}$ \\
HPLC/UV & $0.3 \times 10^{-7}$ & 21 \\
LC/MS & 22
\end{tabular}

either in pharmaceutical dosage forms or in biological fluids. As shown in Table 5 , the proposed method is considered the superior in sensitivity compared with all electroanalytical methods and most of the previously reported chromatographic methods for RS determination. Moreover, PGE provides another advantage of the proposed method, as it is simple, with high electrochemical reactivity, cost effective and more environmentally friendly electrode.

\section{Conclusion}

An electrochemical sensing platform was constructed using rGO and silver nanocomposites to modify pencil graphite electrode. The AgNPs-rGO/PBB bilayered sensor was developed using in situ electro-deposition of rGO and AgNPs hybrid. The modified electrode was characterized using different methods; CV, SWV, LSV, electrochemical impedance, SEM images, FT-IR spectroscopic and Raman spectroscopic methods. The electrocatalytic behavior of the studied electrodes has been further evaluated by calculating the overpotential at current density 10 $\mathrm{mA} \mathrm{cm}{ }^{-2}$ and the Tafel slopes from the fitted Tafel plots. It has been concluded that the in situ electrochemical deposition of AgNPs-rGO over PBB platform enhanced the performance of the studied electrode. This behavior may be attributed to the synergistic effect of the conducting properties of rGO and increasing the surface active area as a result of silver nanocomposites incorporation. The increase in the contacted area of the studied analyte on the electrode surface was confirmed by calculating the surface active area using Randles-Sevcik equation. The obtained electrochemical sensing platform was successfully applied for the ultra-trace determination of RS in pure, pharmaceutical dosage forms and human plasma with detection limit of $2.17 \times 10^{-9} \mathrm{~mol} \mathrm{~L}^{-1}$.

\section{Ethical approval}

The application on human plasma samples was performed in accordance with the Declaration of Helsinki and approved by the Egyptian Network of Research Ethics Committees (ENREC). Informed consents were obtained from human participants of this study.

\section{Conflicts of interest}

The authors report no conflicts of interest.

\section{References}

1 G. Decher and J. Hong, Buildup of ultrathin multilayer films by a self-assembly process, 1 consecutive adsorption of anionic and cationic bipolar amphiphiles on charged surfaces, Makromol. Chem., Macromol. Symp., 1991, 46, 321.

2 N. I. Zaaba, K. L. Foo, U. Hashim, S. J. Tan, W. W. Liu and C. H. Voon, Synthesis of graphene oxide using modified Hummers method: Solvent influence, Procedia Eng., 2017, 184, 469.

3 M. Zidan, R. M. Zawawi, M. Erhayem and A. Salhin, Electrochemical detection of paracetamol using graphene oxide-modified glassy carbon electrode, Int. J. Electrochem. Sci., 2014, 9, 7605.

4 M. Zhou, Y. Wang, Y. Zhai, J. Zhai, W. Ren, F. Wang and S. Dong, Controlled synthesis of large area and patterned electrochemically reduced graphene oxide films, Chem.Eur. J., 2009, 15, 6116.

5 P. Tiwari, A. Kumar and R. Prakash, Electrochemical detection of azidothymidine on modified probes based on chitosan stabilized silver nanoparticles hybrid material, RSC Adv., 2015, 5, 90089.

6 M. R. El-Zahry, A comparative study of sterically and electrostatically stabilized silver nanoparticles for the determination of muscle relaxant tizanidine: Insights of localized surface plasmon resonance, surface enhanced Raman spectroscopy and electrocatalytic activity, Talanta, 2018, 186, 229.

7 A. Luvai, W. Mbagaya, A. S. Hall and J. H. Barth, Rosuvastatin: A review of the pharmacology and clinical effectiveness in cardiovascular disease, Clin. Med. Insights: Cardiol., 2012, 6, 17.

8 C. Stancu and A. Sima, Statins: Mechanism of action and effects, J. Cell. Mol. Med., 2001, 5, 378.

9 M. F. Lima, R. J. Cassella and W. F. Pacheco, Spectrophotometric determination of rosuvastatin in 
pharmaceutical formulations using quinalizarin, Braz. J. Pharm. Sci., 2017, 53, 1.

10 A. A. Ramadan, H. Mandil and N. Alshelhawi, Spectrophotometric determination of rosuvastatin calcium in pure form and pharmaceutical formulation by the oxidation using iodine and formation triiodide complex in acetonitrile, Int. J. Pharm. Pharm. Sci., 2014, 6, 579.

11 T. A. Wani, I. A. Darwish and N. Y. Khalil, Novel microwellbased spectrophotometric assay for the determination of rosuvastatin calcium in its pharmaceutical formulations, Curr. Pharm. Anal., 2013, 9, 54.

12 G. Tuljarani, D. G. Sankar, P. Kadgapathi, R. Suthakaran and B. Satyanarayana, Visible spectrophotometric determination of rosuvastatin in bulk and pharmaceutical formulations, Orient. J. Chem., 2010, 26, 589.

13 R. Cassella, U. Federal, W. Pacheco and U. Federal, Determination of rosuvastatin in urine by spectrofluorimetry after liquid-liquid extraction and derivatization in acidic medium, J. Fluoresc., 2012, 23, 49.

14 I. Süslü, M. C. Elebier and S. Altınoz, Determination of rosuvastatin in pharmaceutical formulations by capillary zone electrophoresis, Chromatographia, 2007, 66, S65.

15 B. G. Chaudhari, N. M. Patel and P. B. Shah, Determination of simvastatin, pravastatin, and rosuvastatin in tablets by HPTLC, Indian J. Pharm. Sci., 2007, 69, 130.

16 S. U. Devi, E. P. Latha, C. V. N. Guptha and P. Ramalingam, Development and validation of HPTLC method for estimation of rosuvastatin calcium in bulk and pharmaceutical dosage forms, Int. J. Pharma Bio Sci., 2011, 2, 134.

17 R. S. Potawale and S. Y. Gabhe, HPTLC method for simultaneous determination of rosuvastatin and fenofibrate in bulk and pharmaceutical formulation, Int. J. Pharm. Pharm. Sci., 2014, 6, 323.

18 N. Karadas-bakirhan, M. Gumustas, B. Uslu and S. A. Ozkan, Simultaneous determination of amlodipine besylate and rosuvastatin calcium in binary mixtures by voltammetric and chromatographic techniques, Ionics, 2015, 22, 277.

19 T. A. Silva, H. Zanin, F. C. Vicentini, E. J. Corat and O. Fatibello-Filho, Electrochemical determination of rosuvastatin calcium in pharmaceutical and human body fluid samples using a composite of vertically aligned carbon nanotubes and graphene oxide as the electrode material, Sens. Actuators, B, 2015, 218, 51.

$20 \mathrm{~S}$. Altnoz and B. Uyar, Electrochemical behaviour and voltammetric determination of rosuvastatin calcium in pharmaceutical preparations using a square-wave voltammetric method, Anal. Methods, 2013, 5, 5709.

21 T. A. Silva, G. F. Pereira, O. Fatibello-Filho, K. I. B. Eguiluz and G. R. Salazar-Banda, Square-wave voltammetric determination of rosuvastatin calcium in pharmaceutical and biological fluid samples using a cathodically pretreated boron-doped diamond electrode, Diamond Relat. Mater., 2015, 58, 103.

22 A. A. Ramadan, H. Mandil and N. Ghazal, Electrochemical behavior and differential pulse polarographic determination of rosuvastatin in pure form and in pharmaceutical preparations using dropping mercury electrode, Int. J. Pharm. Pharm. Sci., 2014, 6, 128.

23 A. A. Ramadan, H. Mandil and N. Ghazal, Differential pulse polarographic behavior and determination of rosuvastatin in pure form and in pharmaceutical preparations using a static mercury drop electrode, Int. J. Pharm. Sci., 2015, 7, 389.

24 A. V. Yegorova, G. A. Fedosenko, I. I. Leonenko, G. V. Maltsev and V. P. Antonovich, Determination of residual amounts of rosuvastatin calcium on the surfaces of pharmaceutical equipment by HPLC and luminescence, J. Anal. Chem., 2016, 71, 126.

25 D. Xu, Z. Ruan, Q. Zhou, H. Yuan and B. Jiang, Quantitative determination of rosuvastatin in human plasma by liquid chromatography with electrospray ionization tandem mass spectrometry, Rapid Commun. Mass Spectrom., 2006, 20, 2369.

26 H. O. Kaila, M. A. Ambasana, R. S. Thakkar, H. T. Saravaia and A. K. Shah, A new improved RP-HPLC method for assay of rosuvastatin calcium in tablets, Indian J. Pharm. Sci., 2010, 72, 592.

27 D. Suares and B. Prabhakar, Stability-indicating assay method for determination of rosuvastatin in nanoformulation and pharmaceutical dosage form by RP-HPLC, Int. J. PharmTech Res., 2016, 9, 265.

28 M. A. Mukthinuthalapati, V. Bukkapatnam, S. Pavan and K. Bandaru, Stability indicating liquid chromatographic method for the simultaneous determination of rosuvastatin and ezetimibe in pharmaceutical formulations, Adv. Pharm. Bull., 2014, 4, 405.

29 B. M. Ishaq, K. V. Prakash, C. H. Kumar, G. U. Rani and G. K. Mohan, Development and validation of LC-MS method for determination of rosuvastatin hydrochloride in human plasma, J. Chem. Pharm. Res., 2010, 2, 324.

30 F. M. Abdel-aal and M. F. B. Ali, Eco-friendly fabricated electrochemical sensor using red cabbage extract for electrochemical determination of dacarbazine with the aid of factorial design approach, J. Electrochem. Soc., 2017, 164, H1053.

31 A. Moradi Golsheikh, N. Huang, H. Lim, R. Zakaria and C. Y. Yin, One-step electrodeposition synthesis of silvernanoparticle-decorated graphene on indium-tin-oxide for enzymeless hydrogen peroxide detection, Carbon, 2013, 62, 405.

32 International Conference on Harmonization (ICH) Topic Q2 (R1): Validation of Analytical Procedure: Text and Methodology, Nov 2005.

33 M. Bera, P. Gupta and P. K. Maji, Facile one-pot synthesis of graphine oxide by sonication assisted mechanochemical approach and its surface chemistry, J. Nanosci. Nanotechnol., 2018, 18, 902.

34 Z. Xu, H. Gao and H. Guoxin, Solution-based synthesis and characterization of a silver nanoparticle-graphene hybrid film, Carbon, 2011, 49, 4731.

35 A. Pajor-Świerzy, T. Kruk and P. Warszyński, Enhancement of the electrocatalytic properties of prussian blue 
containing multilayer films formed by reduced graphene oxide, Colloid Interface Sci. Commun., 2014, 1, 6.

36 J. R. F. Foronda, S. M. R. Cabrera, D. L. Cumpas, P. G. a Villar, J. L. Tan and B. J. V Tongol, Enhanced electrocatalytic activity of $\mathrm{Pt}$ particles supported on reduced graphene oxide/poly (3,4-ethylenedioxythiophene) RGO/PEDOT composite towards ethanol oxidation, $J$. Chem., 2013, 2013, 1.

37 A. J. Bard and L. R. Faulkner, Electrochemical Methods: Fundamentals and Applications, John Wiley \& Sons, New York, 2nd edn, 2001.
38 E. Laviron, General expression of the linear potential sweep voltammogram in the case of diffusion less electrochemical systems, J. Electroanal. Chem., 1979, 101, 19.

39 C. P. Andrieux, F. Gonzalez and J. M. Savéant, Homolytic and heterolytic radical cleavage in the Kolbe reaction: Electrochemical oxidation of aryl methyl carboxylate ions, J. Electroanal. Chem., 2011, 498, 171.

40 M. Reza Ganjali, Highly sensitive voltammetric sensor for determination of ascorbic acid using graphite screen printed electrode modified with $\mathrm{ZnO} / \mathrm{Al}_{2} \mathrm{O}_{3}$ nanocomposite, Int. J. Electrochem. Sci., 2017, 12, 3231. 